\title{
JUEGO Y EDUCACIÓN: reflexiones sobre historia de la recreación en el Uruguay
}

\author{
Rodrigo Píriz ${ }^{1}$ \\ Martín Caldeiro²
}

\section{RESUMEN}

Este trabajo se propone reflexionar sobre la relación entre educación y recreación en dos momentos puntuales del siglo XX en Uruguay, a la vez que producir nuevos discursos para leer la historia de la recreación. Revisión a la luz de las evidencias históricas, que se coloca en oposición a un discurso sobre la recreación fuertemente vinculado al mercado y a la privatización del saber y la experiencia, materializado en determinados monopolios que giran en torno del ámbito de la recreación. Este texto es resultado de un encuentro entre dos investigaciones y sus work progress, que se centran en la historia de la recreación en el Uruguay y en su vínculo con la educación. Se presentan las principales empíreas sobre las que se erigen algunas reflexiones para pensar, los sentidos y las prácticas puestas a funcionar por la educación física en el Uruguay bajo la denominación de recreación o recreativo, así como también, aproximarse someramente a los discursos sobre la educación del cuerpo contenidos en este elemento siempre difuso a la interna del campo de la educación, a saber, la recreación.

Palabras clave: Recreación. Juego. Educación física. Educación del cuerpo. Historia de la educación.

\section{JOGO E EDUCAÇÃO: refleções a sobre história da recreação no Uruguai}

\section{RESUMO}

Este artigo tem como objetivo refletir sobre a relação entre educação e recreação em dois momentos específicos do século XX no Uruguai, e é resultado de um encontro entre duas investigações que enfocam a história da recreação no país e sua ligação com a educação. Partimos de uma revisão histórica que se coloca em oposição a um discurso sobre recreação vinculado ao mercado e à privatização

\footnotetext{
1 Profesor del Departamento de Educación Física, Tiempo Libre y Ocio de la Universidad de la República, Montevideo, Uruguay. Doctorando em Educación en el Programa de PósGraduação em Educação de la Universidade Federal de Santa Catarina (PPGE-UFSC). https://orcid.org/0000-0002-9892-1590. E-mail: piriz.rod@gmail.com

2 Profesor del Departamento de Educación Física, Tiempo Libre y Ocio de la Universidad de la República, Montevideo, Uruguay. Doctorando em Educación en el Programa de Posgrado em Educación de la Universidade Nacional de la Plata (La Plata, Argentina). https://orcid.org/0000-0003-0798-6637. E-mail: martincaldeiroisef@gmail.com
} 
do conhecimento e materializado em certo monopólio que gira em torno do campo da recreação, sobretudo vinculado à sua formação. Apresentamos os principais dados empíricos sobre os quais é possível levantar reflexões sobre os sentidos e as práticas postas em funcionamento pela Educação Física no Uruguai sob a denominação de "recreação" ou "recreativo", abordando brevemente os discursos sobre a educação do corpo contidos nesse elemento sempre difuso do campo da educação e buscando produzir novos discursos para ler a história da recreação no país.

Palavras-chave: Recreação. Jogo. Educação física. Educação do corpo. História da educação.

\title{
PLAY AND EDUCATION: reflections about history of recreation in Uruguay
}

\begin{abstract}
This paper aims to do a reflection about the relationship between education and recreation in two specific moments of the 20th century in Uruguay, and it is the result of a meeting between two researches that focus on the history of recreation in the country and its connection with education. We start from a historical review that opposes a discourse on recreation linked to the market and the privatization of knowledge and materialized in a certain monopoly that revolves around the field of recreation, especially linked to its formation. We present the main empirical data on which it is possible to raise reflections on the meanings and practices put in place by Physical Education in Uruguay under the name of "recreation" or "recreational", briefly addressing the discourses on body education contained in this element always diffuse in the field of education and seeking to produce new discourses to read the history of recreation in the country.
\end{abstract}

Keywords: Recreation. Game. Physical Education. Body education. History of education.

\section{INTRODUCCIÓN}

Entre los estudios sobre historia de la recreación en el Uruguay que pueden ofrecer un punto de partida encontramos los trabajos de Lema (2017; 2016), Vilas (2009) y Lema y Machado (2009). En los tres trabajos se afirma que la recreación en cuanto fenómeno social y cultural, se encuentra estrechamente vinculada al desarrollo de las sociedades modernas y su creciente industrialización3, lo cual no nos dice mucho acerca de las particularidades del caso uruguayo. Estos autores no presentan un

$3 \quad$ Estas ideas también las podemos encontrar en Waichman $(1998 ; 1997)$ y en Elizalde y Gomez (2010). 
posicionamiento que discorde con los estudios más clásicos sobre tiempo libre, recreación, ocio y lazer - la mayoría asociados a un funcionalismo social -, una vez que estos, a saber, son fruto de la sociedad en la que el trabajo asalariado gana centralidad.

Las dificultades que se encuentran para definir la recreación se asocian a que ésta presenta diferentes enfoques en el plano conceptual y que a nivel práctico sus propuestas adquieren formas de lo más variadas. Actividades que refieren a la recreación o lo recreativo están presentes en diferentes ámbitos sociales y culturales, y en la oferta de las más diversas instituciones. En su relación con la educación las vamos a encontrar tanto en el ámbito formal como el no formal, esto es, en escuelas, ONGs, en propuestas destinadas a lo comunitario, así como también en clubes deportivos. Pero hay un ámbito particular que dialoga con aquellos antes nombrados, es el de la educación física. En consecuencia, consideramos importante preguntarse acerca del sentido que adquiere un discurso sobre la recreación y lo recreativo a la interna del campo de la educación física y como se ha relacionado particularmente a la historia de dicha disciplina y de cómo a partir de este discurso se han configurado ciertas formas de educación del cuerpo.

En este sentido, nos proponemos analizar y contrastar dos momentos determinados sobre la vida institucional de la Comisión Nacional de Educación Física (CNEF), en tanto principal órgano estatal vinculado al fomento y desarrollo de la educación física, el deporte y la recreación en el Uruguay4. Por un lado, la producción documental con autoría de Julio J. Rodríguez ${ }^{5}$, correspondiente al período comprendido entre los años 1920 y

\footnotetext{
${ }_{4}^{4}$ Este rol queda adjudicado a la CNEF en ley $N^{\circ} 3.798$ promulgada por el Poder Legislativo el 7 de julio de 1911.

${ }^{5}$ Director técnico de la CNEF desde 1920 hasta 1956, sin duda una de las más influyentes figuras a nivel de la Educación física en la primera mitad del siglo XX en Uruguay. Graduado como Profesor de Educación Física por el Springfield College en 1920. Es autor de varios de los documentos oficiales de la CNEF del período, entre los que se encuentran los planes nacionales de educación física y recreación, informes de la CNEF y el primer proyecto para la preparación de maestros de Educación Física en el Uruguay del año 1921. Se desempeño como profesor de la asignatura recreación entre los años 1939 y 1956 en el "Curso para la Preparación de Profesores de Educación Física" de la CNEF.
} 
1956, entre los que destacamos los documentos "Recreación. Definición e Interpretación" (RODRíGUEZ, 1950), y "Curso de Recreación y Plazas de deportes. Influencia del juego en la salud física y moral del niño" (RODRÍGUEZ, s/f). Rodríguez integró el plantel docente que impartió la formación de profesores de educación física en Uruguay durante un período aproximado de 20 años. Por otro lado, centramos la mirada en el período que se denominará como posdictadura (1985-1990) y particularmente en los programas curriculares de la CNEF, sin duda, los principales documentos producidos por la comisión en materia de políticas en educación física en dicho período.

En la arbitrariedad del recorte y en la dispersión cronológica en cuanto principio metodológico, se constituye un acometimiento en el que confluyen las investigaciones ya desarrolladas y actualmente en curso'. Y es ahí, en esos resultados marginales y empíreas poco exploradas, que toma materialidad dicho trabajo.

\section{RECREACIÓN Y EDUCACIÓN FÍSICA EN LOS ESCRITOS DE JULIO J. RODRÍGUEZ}

Las reflexiones 7 sobre la figura de Julio J. Rodríguez es producto de una investigación más amplia sobre la recreación, el juego y el tiempo libre en el Uruguay en la primera mitad del siglo XX. Investigación que da continuidad a las temáticas desarrolladas en la tesis de maestría titulada $O$ corpo em jogo: a emergência do discurso sobre o jogo no campo da Educação Física no início do século XX no Uruguai (CALDEIRO, 2017). Si bien la investigación es un estudio de los discursos sobre el juego, el autor encuentra referencias a la recreación en una estrecha vinculación al primero, lo que le permite afirmar que la recreación en su estrecho vínculo con la educación física tuvo desde

\footnotetext{
6 Dichas investigaciones se vienen desarrollando en el marco del Programa de Posgrado en Educación de la Universidad Federal de Santa Catarina (Brasil) y del Departamento de Educación Física Tiempo libre y ocio del Instituto Superior de Educación Física de la Universidad de la República (Uruguay).

7 Para ampliar los estudios sobre Rodríguez es ineludible consultar el trabajo de maestría de Paola Dogliotti 2012. Disponible en: https://www.cse.udelar.edu.uy/blog/tesis/cuerpo-ycurriculum-discursividades-en-torno-a-la-formacion-de-docentes-de-educacion-fisica-enuruguay-1874-1948/ (17 de febrero de 2020 última consulta).
} 
sus inicios un enfoque fundamentalmente educativo, y en este entramado, el juego se configuró como el medio principal para el cumplimiento de sus objetivos.

En el caso de la producción de Julio J. Rodríguez son varios los documentos que van a hacer referencia de forma directa a la recreación ${ }^{8}$ y en los que se encuentran tematizaciones sobre sus fundamentos, los sentidos, las formas y los modos de desarrollarla en el Uruguay. Preguntarse acerca de: cuáles eran los principios de la recreación, su contenido educativo, sobre qué postulados propone, qué prácticas y los fundamentos de esas prácticas, es una posibilidad de lectura para dichos documentos. De este modo, comenzaremos analizando el documento "Recreación. Definición e Interpretación" (RODRÍGUEZ, 1950), dicho documento cuenta con cuatro núcleos temáticos que lo estructuran: Las actividades recreativas propiamente dichas, la relación de la recreación con el juego, la relación con el trabajo y la organización de la recreación. En segundo lugar analizaremos el documento denominado "Curso de Recreación y Plazas de deportes. Influencia del juego en la salud física y moral del niño" (RODRÍGUEZ, s/f), estimamos que este material pudo formar parte del curso de profesores de educación física entre los años 1939 y 1959, ya que en dicho período consta que había en la formación un curso con esa misma denominación.

La recreación para Rodríguez (1950) se presenta como un sector de la actividad humana, en términos de unidad con sentido propio, una de las tantas experiencias o esferas sobre las que se organiza y define lo humano. La intención de quien realiza la actividad será la que defina su carácter de recreación, será entonces en la "intención de recrearse que motiva la realización de ciertos actos y la adopción de una especial actitud"

\footnotetext{
8 Entre estos encontramos: "Plan de Acción de la Comisión Nacional de Educación Física y conclusiones que se derivan del mismo" (CNEF, 1923); "Una reseña histórica de la educación física y la recreación en el Uruguay" (CNEF, 1952); "La Educación Física y la Recreación en el Uruguay" (CNEF, 1957); "Curso de Recreación y Plazas de Deportes. Influencia del juego en la salud física y moral del niño (CNEF, s/d); "Recreación. Definición e interpretación" (CNEF, 1950); "Recreación" (CNEF, 1950) y "CNEF. Dirección Técnica. Recreación" (CNEF, 1956).
}

Revista Exitus, Santarém/PA, Vol. 10, p. 01-16, e020024, 2020. 
(Rodríguez, 1950, p. 2) definiendo el carácter subjetivo de lo que pueda ser llamado recreación. Autodeterminación ésta, que también está presente en las definiciones del ocio para J. Dumazedier, siendo uno de los puntos más obscurantistas para entender el fenómeno, ya que la dimensión de lo subjetivo y el significado que adquiere para quien lo realiza es lo que lo determina (MUNNÉ, 2017), casi anulando toda posibilidad de objetivación.

J. Rodríguez en el documento de 1950 define al hombre como "un conjunto unitario de actos", organizados por sectores de actividad: "trabajo, vida política, recreación y deporte" (RODRíGUEZ, 1950, p.1). De esta forma, la recreación queda diferenciada de otros campos de actividad y se constituye por oposición a las actividades serias de la vida, del "campo fundamental de actividades, de las actividades serias y esenciales" (RODRÍGUEZ, 1950, pp. 2-3), configurándose en torno de la diversión y el relajamiento. En tal sentido, el tiempo de recreación requiere distinguirse de las actividades serias y esenciales de la vida, y orientarse a realizar el "[...] deseo de aventura y de juego [...] sus ansias de gozo, en un ámbito libre de tensiones y exigencias" (RODRÍGUEZ, 1950, p. 2). El juego inscripto en este campo, se funcionaliza por la oposición necesaria a las actividades serias, para el autor el juego es pura despreocupación, es y debe serlo, de lo contrario no cumpliría con las ansias de gozo.

La esfera de la recreación es el campo de la realización [...] donde pueden darse experiencias siempre nuevas de vida fresca y natural, la acción espontánea y desinteresada, la liberación de la fantasía, el cumplimiento del deseo de aventura y de juego en un entorno alegre y ligero (RODRÍGUEZ, 1950, pp. 2-3).

La ilusión propia del siglo XVIII se hace presente en esta cita, aquella que reconocía en el ser humano una especie de naturaleza, separada de la vida social que contamina la "esencia" humana. La recreación y el juego particularmente, se van configurando por un discurso que les asigna un carácter de ligereza y alegría. La recreación se presenta como un campo escindido de lo laboral, distanciado de la economía y de las instancias serias de la vida. Pero en realidad queda inscripta en un funcionamiento 
compensatorio del tiempo de trabajo, funcional a una lógica de mercado, ya que su función es equilibrar la vida.

Pero además la esfera de la recreación es el mundo sin exigencias y sin tensión, donde es posible apartarse momentáneamente de ese otro mundo serio, tenso y exigente y sumergirse despreocupada y desprevenidamente. La recreación es, pues, en primer lugar, un factor de integración indispensable para la plenitud y total realización del hombre y además un elemento de equilibrio de la vida (RODRÍGUEZ, 1950, p.3).

Para J. Rodríguez "La recreación se logra, generalmente, por medio de actividades, pero ni se identifica ni está determinada por ellas" (1950, p.5). Esta afirmación es interesante para comprender qué es la recreación. Lo central se ubica en la actitud con la que el sujeto la practica. En este sentido, la recreación no es una práctica determinada sino más bien una actitud del ser, que le otorga al tiempo, sentido.

Porque no requiere ineludiblemente actividades para cumplirse una actitud remplaza en ciertos casos al hacer, y lo que es más importante, porque una actividad, en cuanto realización de determinada forma, puede darse en muy diferentes sectores y poseer muy diferentes significados (deporte, trabajo, recreación, política, etc). La forma o apariencia externa no indica siempre con certeza el sentido y, por lo tanto, el sector al que pertenece. [...] la intención del hombre informa las acciones con un especial sentido y las endereza a determinado sector. Es la intención la que establece que jugar al football sea para el jugador profesional trabajo, deporte para el jugador olímpico y recreación para el que juega por placer (RODRÍGUEZ, 1950, p.5).

El tiempo se fragmenta y se individualiza, ya que el sentido lo define cada individuo en función de su intencionalidad en consonancia con su actitud. La racionalidad y la voluntad determinan las actitudes y los sentimientos. Esto se fortalece en la modernidad y se vuelve condición para que emerja la recreación.

Por otro lado, el documento titulado "Curso de Recreación y Plazas de Deportes. Influencia del juego en la salud física y moral del niño" (Rodríguez, s/f) muestra el enfoque educativo asignado a la recreación y la perspectiva a adoptar desde el punto de vista conceptual, a saber, una "ecléctica" (Rodríguez, s.f, p. 1). El entramado discursivo de Rodríguez expresa que la 
salud del niño "depende del buen estado de sus órganos y de su funcionamiento normal y eficiente" y que la misión de los que tienen a cargo la educación del niño es asegurar su crecimiento normal (Rodríguez, s.f, p.2). La educación física, entonces, a través del desarrollo de los juegos en el niño, tiene como principal cometido su educación; lo orgánico cobra centralidad estableciéndose por parámetros, criterios de normalidad, procedimientos de carácter biopolítico. Mediante la normalización del juego "la actividad necesaria y moderada de los órganos, especialmente de aquellos que se hallan en el período de crecimiento rápido tiende al perfeccionamiento de su estructura y eficiencia funcional" (Rodríguez, s.f, p. 3). Así el juego y la recreación se fundamentan bajo un sustrato biológico articulando la dimensión pedagógica y psicológica. Esta última se manifiesta al determinar los juegos para cada una de las etapas evolutivas, y al diferenciar el juego en la etapa infantil y la recreación en la adultez. El carácter educativo del juego se viabiliza no solo a través de la articulación con lo orgánico del cuerpo, sino que al analizarlo desde una perspectiva del orden de lo moral se afirma que:

[...] el campo de juego es una escuela donde se cultiva el carácter [...] la responsabilidad total, corresponde al maestro o al director de juegos. Este es el que debe moldear el carácter de los niños, pues el campo de juego, le ofrece la oportunidad para practicar esas enseñanzas (RODRÍGUEZ, s.f, p. 4).

A partir de lo analizado hasta el momento, podemos decir que el juego es presentado como aquel medio adecuado para introducir al niño en ciertos parámetros de comportamiento. El campo de juego dice Rodríguez "es una escuela donde se cultiva el carácter" (Rodriguéz, s/f, p. XX). Es evidente que la figura del adulto, maestro o director de juegos según el autor, tiene la responsabilidad educativa que se inscribe en las instancias recreativas. De este modo, podemos afirmar que la recreación tiene desde la primera mitad del siglo XX en Uruguay un carácter educativo y se gesta en estrecho vínculo con la educación física, presentándose como una forma particular de educación del cuerpo. 


\section{Educación física, recreación y posdictadura: Los programas curriculares de la CNEF}

En el trabajo de Píriz (2018), podemos ver un desarrollo temático que se centró en el análisis de los discursos sobre la recreación presentes en las políticas en educación física desarrolladas por el Estado uruguayo, en el periodo posterior (1985-1990) a la última dictadura cívico-militar (1973-1985). Pensar dichas políticas en tanto políticas del cuerpo, en su estrecha relación con las estrategias modernas de gobierno, constituye la idea general que guió dicha investigación. Es así que se identificaron los elementos constitutivos de lo que se presentó como recreación y recreativo - tanto a nivel conceptual como de las prácticas que se presentaban bajo ese rótulo a la interna de la CNEF, entendiendo a dicho órgano como el principal ámbito en donde estas prácticas eran puestas a funcionar y en donde a su vez encontraban un espacio de legitimación en la órbita estatal.

Partiendo del análisis de los documentos consultados?, fue posible identificar y describir prácticas vinculadas al tiempo libre de la población, que a su vez eran solidarias al espíritu de una época caracterizada por un Estado abocado al restablecimiento de la democracia.

En este sentido, se realizó un análisis conceptual de los diferentes elementos que constituían lo que se consideró como una definición de la recreación y lo recreativo a la interna de la CNEF. Así, fue posible identificar elementos conceptuales que permitieron pensar para el caso uruguayo la constitución de un enfoque de la educación física, que a la vez que explicita su cualidad lúdica - elemento constitutivo de la educación física en tanto forma de educación del cuerpo que viene a sustituir a la gimnástica según Crisorio (2007) - por medio de su característica recreativa - aparentemente en detrimento de otros elementos que constituyen su matriz epistemológica-,

\footnotetext{
9 Son cinco los documentos principales utilizados en dicha investigación: "Programa de Recreación" (CNEF, 1988a), "Programa de Educación Deportiva" (CNEF, 1988b), "Programa de Educación Física Nivel Inicial" (CNEF, 1988c), "Programa de Educación Física Nivel Médio" (CNEF, 1988d) y "El Deporte, la Educación Física y la Recreación" (CNEF, 1990).
} 
va a instaurar una separación con lo que se empieza a constituir como campo de la recreación en el Uruguay ${ }^{10}$.

A partir de los documentos señalados, en la investigación (PÍRIZ, 2018), es posible identificar una apropiación por parte de la CNEF de las diferentes tematizaciones y abordajes - tanto conceptuales como prácticos presentes en los principales enfoques sobre recreación que se desarrollaron en Uruguay durante el siglo XX'1 .

La recreación va a estar presente en los cuatro programas de educación física propuestos por la CNEF en el año 1988. Ya sea en cuanto contenido específico o como cualidad y abordaje que debían presentar algunas prácticas históricamente vinculadas al campo de la educación física - en este caso resaltamos las danzas, los deportes y la gimnasia -, bajo la forma de lo recreativo. Esta presencia no es menor, en relación al programa de Educación Deportiva (CNEF, 1988b), en donde la recreación es la forma como deben ser abordadas las prácticas deportivas en sus fases iniciales. A su vez, lo recreativo, aparece como el carácter con el que debe ser practicado el deporte por quienes serán adeptos, no vinculándose a los ámbitos de alto rendimiento, a lo que se esboza un modelo de deporte recreativo, el que sería ajeno a la propaganda, a la comercialización y a la instrumentalización política. Esta forma del deporte tiene funciones de ocio activo, higiene-salud, desarrollo biológico, esparcimiento, educación, pausa en el tecnicismo, relación social, superación, el que en alguna medida se podría identificar con un carácter autotélico y hedonístico, que pongan en evidencia su carácter lúdico, vinculado más al juego y al contacto del hombre con la cultura (CNEF, 1988b; PIRIZ, 2018).

En el caso de los programas de Educación Física Nivel Inicial (CNEF, 1988c) y de Educación Física Nivel Medio (CNEF, 1988 d), lo recreativo

\footnotetext{
${ }^{10}$ Este va a comenzar a dibujarse a partir de un ámbito - o varios mejor dicho - de actuación determinado y la constitución de diferentes niveles de profesionalización y formación. Igualmente consideramos esto no representa un desplazamiento de los profesionales de la educación física, como algunos autores afirman.

11 Una lectura detallada sobre los enfoques en recreación que se desarrollan en el siglo XX en el Uruguay se encuentran en: "Más acá de una historia de la recreació" (PÍRIZ, 2018, pp. 29- 62).
} 
refiere principalmente a la educación para el tiempo libre y para el juego, siempre refiriéndose a la buena utilización en el caso de lo primero y al correcto desarrollo del niño en el caso de lo segundo. Así, la recreación y las actividades recreativas ofician como auxiliares de la educación física y de las instituciones educativas ampliando su espectro de acción sobre el niño, el joven y la comunidad. En este sentido, uno de los principales objetivos que comparten ambos programas es el de "Demostrar una inserción acorde a las condiciones del medio ambiente, tanto humano como material, a través de la Educación Física, la Recreación y el Deporte. Apoyar y co participar en las tareas del grupo, de la escuela, del centro Recreativo - Deportivo y de la comunidad" (PÍRIZ, 2018, p. 80).

En el caso del Programa de Recreación (CNEF, 1988a) este, es sin duda uno de los documentos más importantes del Estado uruguayo en la materia del período en cuestión. Es el que presentará las principales definiciones y fundamentos sobre recreación y las actividades recreativas por parte de la CNEF, como también cuáles son los objetivos de este tipo de prácticas. De forma sintética podemos decir que los objetivos de dicho programa refieren a la educación para el uso del tiempo libre, direccionado a la actividad física como forma de satisfacer necesidades e intereses; generar las condiciones para que la población pueda acceder al recrearse por medio de prácticas deportivas; un desarrollo de la recreación como práctica específica; mantener y fomentar las costumbres y tradiciones; ofrecer un vasto abanico de actividades a la población; combatir el sedentarismo y sus efectos en la experiencia motriz del individuo y en función a esto generar en la población una conciencia del tiempo libre en la que por medio de la recreación se promueva la formación integral y permanente del individuo (CNEF, 1988a: 5). La búsqueda del placer en las actividades a realizar en el marco de lo que se denomina como recreación va a estar en total consonancia con la idea de la búsqueda de la emoción en el ocio de Elías y Dunning (1992). En este sentido, 
[...] la recreación se va a presentar como una búsqueda de emociones que se diferencien de la vida cotidiana por medio de espacios que constituyen una estrategia socialmente establecida y aprobada, que presenta la posibilidad de expresión de las emociones, en una suerte de mimetización de la vida ordinaria (PÍRIZ, 2018, p. 146).

Pero sobre todo, uno de los elementos particulares de este último programa será la definición y clasificación de los tiempos y espacios sociales que determinen los ámbitos para el desarrollo de la recreación. Es a partir de la definición de los tiempos sociales de Frederic Munné (2017) y de los espacios sociales destinados al tiempo libre de la Arq. Graciela Schidmit12 que se desarrollan dichas temáticas (CNEF, 1988a).

Estos elementos son las evidencias que permitieron realizar un análisis conceptual sobre la relación recreación y educación del cuerpo en el período señalado:
Si en un primer nivel en los documentos aparecían nociones como cuerpo, lúdico y tiempo libre, en tanto motor del análisis, en un segundo nivel y más específicamente sobre el concepto de recreación, son las nociones de tiempo libre, libre elección y búsqueda del placer, las que van a aparecer como elementos constantes en los diferentes abordajes sobre la temática (PÍRIZ, 2018, p. 146).

Algo que es posible identificar a partir de los programas de la CNEF del año 1988 es, un territorio caracterizado por cierta heterogeneidad e indefinición en relación a los discursos sobre la recreación y lo recreativo. La centralidad va a estar en la compensación y complementariedad en relación a la esfera del tiempo social que representa lo serio - el trabajo -, traduciéndose en una preocupación por el uso apropiado del tiempo libre, en donde la característica que le asigna particularidad a sus actividades está representada por su carácter lúdico, articulado con preocupaciones a

\footnotetext{
12 La Arq. Graciela Schidmit, es una de las fundadoras de la Comisión de Mujeres Arquitectas de la ciudad de Rosario, Argentina. Dicho grupo tiene entre sus preocupación la configuración arquitectónica y urbanística de los espacios públicos, declarando que "Nuestro aporte es proporcionar una reflexión crítica y diseñar los procesos de intervención urbana, permitiendo la apropiación del espacio sin exclusiones de sexos, clases sociales, edades, discapacidades, herencias culturales o credos" (Pagina 12 del 29 de mayo de 2009). Cómo la Arq Schmidt llega a formar parte de los materiales con los que se elabora el programa de recreación es hasta ahora una incognita que merece ser atendida.
} 
nivel de desarrollo humano y la democratización de la cultura. Entonces, en dicha indeterminación es que la CNEF realiza una síntesis conciliadora que integra los diferentes enfoques sobre la recreación, no sin intentar formular un discurso propio en el que se puede leer un higienismo renovado, en donde la preocupación central sigue siendo el cuerpo productivo, pero ahora con el imperativo del goce como base justificativa. Discursos en donde lo que parecería siempre estar en juego es la representación del cuerpo y su educación.

\section{CONSIDERACIONES FINALES}

Analizar el derrotero de la recreación en el Uruguay y su vínculo con el ámbito educativo - ya sea formal o no formal - sin tener en cuenta su vínculo histórico con la educación física es un recorrido absolutamente incompleto. Los documentos analizados en este texto y otros movilizados en las tesis que sirven de antecedentes a este trabajo dan cuenta de este estrecho vínculo entre recreación y educación física. Si como se afirma en Píriz (2018), la recreación, o más precisamente lo recreativo, se introduce en Uruguay a través de la Young Men's Christian Association en su llegada institucional en 1909 y aparece como uno de los objetivos centrales para la CNEF en 1923, bajo el nombre de recreación física (CNEF, 1923). A modo de hipótesis planteamos que la recreación comienza a cobrar autonomía hacia la década de los 40, momento en el cual aparecen cursos específicos sobre Recreación en los ámbitos de formación de profesionales de la educación física.

A partir de los documentos analizados se identifica a J.J Rodríguez como uno de los primeros referentes sobre la temática y responsable de los cursos implementados a mediados del siglo XX para la formación de profesores. Es en este período que aparece explícitamente en el documento del plan de acción de la CNEF de 1949 el concepto tiempo libre, elemento central para pensar la recreación como práctica y su vinculo con la educación. A partir de ese momento, recreación y tiempo libre conforman un binomio a la interna del campo de la educación física. Vinculo que tiene 
continuidad hasta hoy en día, o por lo menos, hasta finales del siglo XX, como demuestran los documentos analizados.

Encontramos en los dos momentos presentados un discurso sobre la recreación que parecería constituir o más bien definir el carácter de un modo de experimentar una actividad. En la condición de liberado de obligaciones el sujeto accede por medio de la recreación o lo recreativo a un tipo de experiencia en donde adquiere autonomía, según continuidades que aparecen en los discursos analizados. Experiencia que se justifica en la búsqueda del placer, es decir, ya no se trata de una distensión y descanso que permita una óptima recuperación para retornar a la jornada laboral, ahora se trata del disfrute, esta es la forma que toma la compensación de lo serio.

De este modo, la recreación en cuanto actividad específica, adopta para sí las más variadas actividades, pero siempre teniendo en el centro la idea de disfrute, libre elección y sentimiento de libertad, desdibujándose en la vivencia personal en cuanto actividad específica. Ya sea en la incorporación del deporte, de las actividades escénicas, la música, la plástica, y las más variadas actividades que podamos imaginar, lo que aparece a partir de los documentos analizados como su contenido constitutivo, es el juego, impregnando el resto de las actividades con sus características más fundantes.

En este sentido, podemos arriesgar la hipótesis de que el imperativo por detrás de todo esto, es el del goce del tiempo libre. Para esto está la recreación, que mediante las diferentes prácticas mencionadas anteriormente, al extraerles su sentido más serio y dedicación casi que profesional, nos hace recrearnos. Ya no es un cuerpo que produce y que debe reconstituir su fuerza de trabajo, ahora se trata de un cuerpo que consume y es consumido en los ámbitos de no trabajo. Subjetividad que es necesario formar y a la que los ámbitos educativos no serán indiferentes. Nos encontramos ante un ciclo de consumo del tiempo libre y la cultura, que toma la forma de un comportamiento compulsivo en donde el juego se presenta como su herramienta principal. Comportamiento que bajo la 
metáfora de lo recreativo, y la pretensión de presentarse como un fin sin utilidad, simplemente esconde la fidelidad eterna a su contrario - el trabajo allí donde el juego se pervierte y la cultura pierde toda posibilidad de pensar la alienación.

\section{REFERENCIAS}

Caldeiro, M. O corpo em jogo: a emergência do discurso sobre o jogo no campo da educação física no começo do século XX no Uruguai.

Dissertação (Maestría em Educação) - Universidade Federal de Santa Catarina, Florianópolis/SC, 2017.

CNEF. Plan de Acción. Montevideo: Latina, 1923.

CNEF. Plan de Acción. Montevideo: CNEF, 1949.

CNEF. Programa de Recreación. Montevideo: Nueva Impresora Piscis, 1988a.

CNEF. Programa de Educación Deportiva. Montevideo: Nueva Impresora Piscis, 1988b.

CNEF. Programa de Educación Física. Nivel Inicial. Montevideo: Nueva Impresora Piscis, 1988c.

CNEF. Programa de Educación Física. Nivel Medio. Montevideo: Nueva Impresora Piscis, 1988d.

CNEF. El Deporte, la Educación Física y la Recreación. Montevideo: CNEF, 1990.

Elias, N. Dunning, E. Deporte y ocio en el proceso de la civilización. Buenos Aires: FCE, 1992.

CRISORIO, R. Educación física y biopolítica. TEMAS \& MATIZES, n. 11 PRIMEIRO SEMESTRE, p. 67-78, 2007.

GOMES, C.; ELIZALDE, R. Ocio y recreación en América Latina: conceptos, abordajes y posibilidades de resignificación. Polis [En línea], n. 26, 2010.

LEMA, R; MACHADO, L. Tiempo libre y recreación en Uruguay: la construcción de un enfoque lúdico y educativo. In: GOMES, C. (Org.). Lazer na America Latina: Tiempo libre, ocio y recreación en Latinoamérica. Belo Horizonte: UFMG, 2009.

MUNNÉ, F. Psicosociología del tiempo libre un enfque crítico. Buenos Aires: Editores Asociados, 2017. 
PíRIZ, R. (2018). Corpo, política e lazer: a exemplo do processo de redemocratização no Uruguai (1985-1990). Dissertação (Maestría em Educação) - Universidade Federal de Santa Catarina, Florianópolis/SC, 2017.

RODRÍGUEZ, J. Curso de Recreación y Plazas de Deportes. Influencia del juego en la salud física y moral del niño. Montevideo: Archivo del Centro de Memoria de Educación Física (CEMEF). ISEF, Udelar, s/f.

RODRÍGUEZ, J. Recreación. Definición e interpretación. Montevide: Archivo del Centro de Memoria de Educación Física. (CEMEF). ISEF, Udelar, 1950.

VILAS, F. Recreación en el Uruguay: historia, trayectos, concepciones y modelos. In: GOMES, C. (Org.). Lazer na America Latina: Tiempo libre, ocio y recreación en Latinoamérica. Belo Horizonte: UFMG, 2009. p.188- 201.

WAICHMAN, P. Tempo livre e recreação: um desafio pedagógico. Campinas: Papirus, 1997.

WAICHMAN, P. Acerca de los enfoques en recreación. V Congreso Nacional de Recreación Coldeportes Caldas / Universidad de Caldas / FUNLIBRE, 1998.

Recebido em: 10 de novembro de 2019

Aprovado em: 27 de fevereiro de 2020

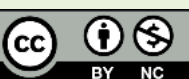

